\title{
Escenas contemporáneas de la educación sexual en Latinoamérica: Una lectura en clave feminista
}

\section{Educación sexual en América Latina: recorrido sobre sus huellas}

La inclusión de temáticas en torno a la sexualidad en las políticas públicas educativas ha sido un campo de debate abierto y explícito en los últimos tiempos en América Latina y el Caribe. Específicamente al analizar el estado actual legislativo y con ello de producción de políticas públicas en este campo se observa la presencia de leyes específicas, programas y la producción de documentos curriculares de diverso orden que apuntalan a su implementación. En trabajos anteriores (Baez, 2015; Baez y González del Cerro, 2015) hemos relevado la formulación de estas políticas en la región observando un proceso de actualización en materia de derechos humanos vinculados a las temáticas de ciudadanía sexual. No obstante, al afinar la mirada en los procesos singulares a nivel nacional rápidamente encontramos un continente donde se comparten heterogéneas (y desiguales) realidades que en los últimos tiempos se han encrudecido por un contexto marcado por el cambio de signo político de los gobiernos. ${ }^{1}$

El giro neoliberal/neoconservador sitúa hoy esta producción de políticas públicas bajo un conjunto de nuevas interpelaciones y orientaciones de sentido. ${ }^{2}$ Los procesos de democratización pos dictadura y la instalación de los derechos humanos en general, incluyendo los derechos sexuales, así como el compromiso de las luchas feministas en politizar el "mundo privado" ha traccionado las lógicas hegemónicas del ordenamiento religioso y/o biomédico (Brown, 2007). Sin embargo, resulta imposible negar, el legado más crudo y resistente que ha dejado la colonización de estas tierras: la colonización de los cuerpos bajo la doctrina católica, particularmente el cuerpo de las mujeres. El poder religioso hoy se actualiza y diversifica en iglesias evangélicas y ocupando en simultaneidad distintas posiciones: dueños de medios de comunicación, ocupando franjas horarias televisivas o cargos legislativos.

En este escenario, la inclusión de la educación sexual alcanzó cierto acuerdo en la región en la década del 70 en una trama de formulación de políticas públicas donde los Estados Nacionales desarrollistas, los Organismos internacionales, los sectores eclesiales y el Movimiento Feminista y de Mujeres, participaron habilitando una agenda pública de género (Baez, 2015). Con demandas diferentes y en posiciones de poder desiguales estos actores configuraron una trama de sentidos que permean la discusión contemporánea.

La clave de intervención política desarrollista ofreció a la temática un campo de acción regional fecundo en el que, la expansión de los sistemas educativos y la necesidad de
* Doctora en Ciencias de la Educación por la FFyL-UBA, investigadora del IICE-CONICET, docente de Investigación educacional II y Abordajes socioeducativos de las problemáticas de género y sexualidades. / baez.jesica@gmail.com
1. En trabajos anteriores -ya citadoshemos analizado por ejemplo la formulación de políticas del campo en Paraguay y Brasil. En estos países se han producido discusiones públicas frente a la implementación de políticas de educación sexual con perspectiva de género que coincidieron con reconfiguraciones político partidarias en cámaras legislativas. para una may profundizislativas. Para una mayor profundización de as controversias generadas en torno a la puesta en marcha del Programa Escuelas sin Homofobia: Maio, E. R. y Batista de Oliveira Júnior, I. (2015). Diversidad sexual y homofobia: la cultura desagendarla en las políticas públicas educativas. En Praxis educative, vol. 10 ( $\left.n^{\circ} 1\right)$, pp. 35 -53. doi: educative, vol. 10 ( $n^{\circ}$ 1), pp. 35-53. doi:
$<10.5212$ /PraxEduc.v.10i1.0002>. Y $<10.5212 /$ PraxEduc.v.10i1.0002>. Y
Batista de Oliveira Júnior, I. (2016). Kit de combate a homofobia do MEC: a polemização em torno dos recursos audiovisuais. En Revista HISTEDBR On-line, ( ${ }^{\circ} 70$ ), pp. 319-334

2. Lo expuesto aquí son resultado del análisis de una serie de fuentes: 1. relevamiento de leyes en la región, 2. relevamiento de documentos de programas de educación sexual en la región, 3. relevamiento de documentos curriculares. La construcción del corpus empírico a su vez se nutrió del diálogo con investigadorxs especializadxs de la región. 
formación de recursos humanos que habilitaron el despegue de los países clasificados como tercermundistas, se construyeran como argumentos sólidos. Las políticas de población, y particularmente, la posibilidad de planificación familiar se tornaron una de las variables centrales en el marco de políticas desarrollistas, cobraron un lugar preponderante en la agenda regional. El concepto de "Educación en población" puesto en juego en el Plan de Acción Mundial sobre Población (PAMP), aprobado en Bucarest en 1974, da ejemplo de ello (Baez, 2015). La región también se vio interpelada por acciones eclesiales, entre las que cabe destacar la encíclica Humanae Vitae (1968) donde los sectores hegemónicos de la Iglesia católica apostólica romana plasmaron sus directrices en torno a la preocupación por la "degradación moral" que implica el uso de métodos anticonceptivos y la presencia del Estado en su regulación (Baez, 2015). Las repercusiones de este documento abrió el debate de posiciones divergentes en el seno eclesial: mientras para algunos funcionarios, intelectuales y episcopados consolidó el valor de la paternidad responsable en el marco del matrimonio monogámico-heterosexual y el objetivo de evitar los abortos; para otros fue una apertura a construir miradas alternativas fuera o dentro de la estructura religiosa (Felitti, 2012).

El avance biotecnológico alcanzado con la llegada al mercado de la pastilla anticonceptiva (el 11 de mayo de 1960 se aprobó la venta de Enovid, el primer anticonceptivo oral en EE.UU, iniciando su comercialización el 18 de agosto del mismo año) motorizó estos debates, también fuertemente nutridos por las voces de feministas. La consigna feminista "lo personal es político" apuntó a interpelar el estatus privado de la sexualidad poniendo en tensión qué temas son los que se constituyen como tales y cuáles como asuntos públicos. Los sentidos de la publicidad y privacidad delimitan el campo de deliberación, y con ello organizan el listado de temas que son parte de la agenda política (Fraser, 2006). En esta dirección, el feminismo en América Latina en tanto colectivo de pensamiento y práctica crítica ha sido precursor de la desconstrucción y confrontación de las hegemonías patriarcales abriendo los interrogantes sobre lo instalado e imaginando caminos alternativos (Carosio, 2014).

La década de los 80, actualizó los debates en torno a la educación sexual a nivel mundial con la aparición de "la epidemia del VIH-sida" y posteriormente, con la problematización de los embarazos en la adolescencia (Morgade, 2006). Ambos fenómenos fueron comprendidos por algunos sectores hegemónicos como "emergencias" que desplegaron un escenario de muerte y temor, junto a la proliferación de mitos y metáforas a su alrededor (Sontag, 2003) así como de "escenarios trágicos de pobreza" para el caso de los embarazos. En este contexto, el acento en la prevención cobró relevancia y el enfoque biomédico tomó las riendas del asunto. No fue, sino hasta el 2008, que mediante la declaración "Prevenir con Educación" en el marco de la XVII Conferencia Internacional de sida en la ciudad de México, que se recuperó y organizó, en la modalidad de un pacto internacional, la necesidad de educación sexual en clave de prevención. La educación sexual es presentada desde una perspectiva basada en los derechos humanos y en el respeto a los valores de una sociedad plural y democrática en la que las familias y las comunidades se desarrollan plenamente.

La llegada del nuevo milenio renovó el escenario de "emergencia" con la problematización de la violencia. Las formas de abordaje de esta temática en las políticas de educación sexual adquiere diversos sentidos. Mientras en algunos países la violencia es comprendida en términos individuales y como efecto no deseado del ejercicio de la sexualidad, en sectores minoritarios avanza un concepto la noción de violencia de género en clave de las relaciones de poder. El tratamiento de estos contenidos abre una serie de interrogante respecto de cómo estos temas refuerzan o no la vivencia de la sexualidad como amenazante, y en qué lugar se ubica a quiénes son víctimas de la misma (Morgade, 2011). En otras palabras, víctimas victimizadas o víctimas empoderadas. Estos interrogantes acontecen en un escenario en el que el movimiento "Ni 
una menos" se han extendido en la región actualizando las agendas del feminismo en torno a la denuncia de la violencia machista.

\section{Escenas pedagógicas contemporáneas}

Del mapa descripto en la sección anterior, quisiera detenerme en tres escenas pedagógicas de la región a fin de analizar las tramas singulares de su configuración y las interpelaciones que se despliegan. En cada una de estas acciones se concentran, articulan y/o pujan nudos de sentidos que enseñan socialmente al territorio, sus sujetos y sus intervenciones. Este "enseñar" adquiere diversas tonalidades en cada una de los recortes propuestos. Mientras que en algunos se tiende a la normalización -es decir, se busca restablecer, reforzar, fundamentar lo normal como producto de la naturalidad-, en otros casos se tiende a irrumpir, disentir y/o proponer otro encuadre. A su vez, la complejidad de estas escenas, nos enfrente a construir una mirada que se pose sobre lo multidimensional, los intersticios y las pocas certezas.

\section{a. Ni una menos}

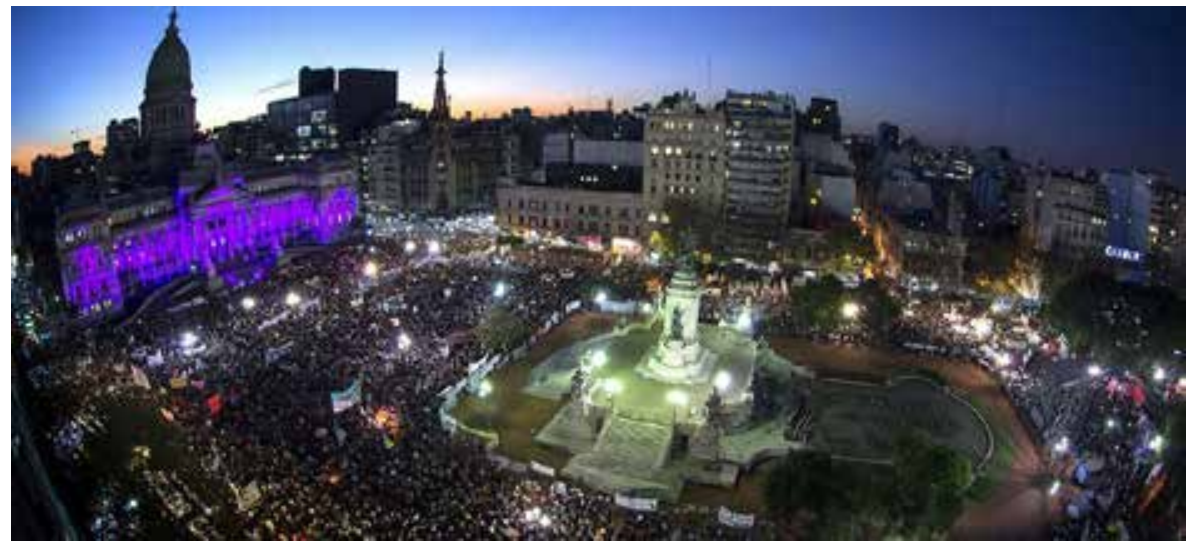

Figura 1. Marcha Ni una menos en la Plaza de los Congresos, Ciudad de Buenos Aires, Argentina. Fecha: 3 de junio de 2015. Fuente: <http://niunamenos.com.ar>.

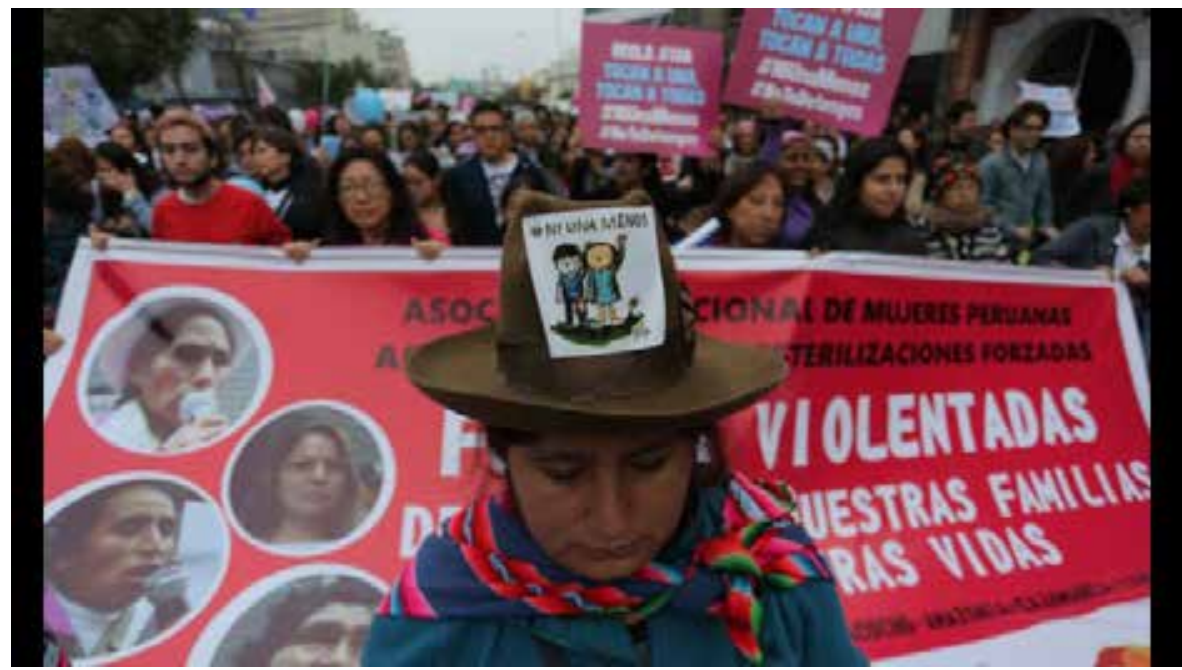

Figura 2. Marcha Ni una menos en Lima Perú. Fecha: 13 de agosto de 2016. Fuente: David Huamaní para el diario La República en <http://larepublica.pe/sociedad/793932-niunamenos-hizo-11-pedidos-para-poner-fin-la-violencia-de-genero>. 
3. Ilustrador argentino que realizóla ilustración que se observa en la Figura 2.
El movimiento "Ni una menos" alcanzó su primer expresión pública mediante la realización de marchas que acontecieron a lo largo del territorio argentino el 3 de junio de 2015. La Figura 1 ilustra la más numerosa de ellas frente al Congreso Nacional ubicado en la Ciudad de Buenos Aires. Con la consigna "Basta de feminicidios", denunciando la muerte de una mujer cada 30 horas, un grupo de periodistas, activistas y artistas convocó al encuentro en las calles. La cita volvió a reiterarse cada año, con diversos énfasis; en el 2016 con "Viva nos queremos", y en el 2017 con "Basta de violencia machista y complicidad estatal". Cada nueva edición, amplió las bases de convocatoria no solo en términos numéricos sino también, en alianzas de trabajo entre organizaciones y consolidando una fuerte estrategia mediática que implicó no solo la cobertura de los hechos sino también aspiró a instalar la temática en la agenda comunicacional. El movimiento, con correr de los meses, construyó vínculos con otros países extendiéndose a la región la consigna. La Figura 2 da cuenta del cruce de fronteras, abriendo interrogantes sobre los alcances y desafíos. En la figura se observa una chola con sombrero y un awayu sobre sus hombros. El sombrero lleva una ilustración realizada por Liniers ${ }^{3}$ en Argentina popularizada en las marchas del país. El pliego de demandas peruanas condensa once puntos que se centran en estrategias de acción frente a "las víctimas de violencia".

Esta primera escena extendida en dos fotografías nos brinda pistas para ampliar la compresión de la organización contemporánea de parte del feminismo. Específicamente me interesa analizar los encuentros, distancias y paradojas que estos movimientos realizan al campo educativo: ¿Qué interpelaciones realizan a los ministerios de educación? ¿Qué posiciones despliegan en torno a la Educación Sexual? ¿Qué estrategias de construcción con otrxs desarrollan?

La educación sexual, tanto en Argentina como en Perú, aparece entre las demandas centrales.

8. Implementar una currícula escolar que promueva "el enfoque de género, la igualdad y la educación sexual integral." 4

4. Garantizar y profundizar la Educación Sexual Integral en todos los niveles educativos, para formar en la igualdad y en una vida libre de discriminación y violencia machista. Sensibilizar y capacitar a docentes y directivos."

Ahora bien, ambas demandas entran en diálogo con la formulación de políticas públicas ya existentes. Desde el 2006 en Argentina por medio de la Ley 26150 que crea el Programa Nacional de Educación Sexual Integral, mientras que en Perú no existe una ley sino una serie de lineamientos curriculares que en distintas oportunidades incluyeron temáticas de educación sexual. En 2008 se consolidaron los lineamientos educativos y orientaciones pedagógicas para la educación sexual integral ${ }^{6}$ que luego, en $2016^{7}$ fueron actualizados. La inclusión del "enfoque de género" inició una campaña conservadora por parte de las iglesias evangélicas y católicas que finalmente avanzó en cambios curriculares en el 2017. ${ }^{89}$ En este proceso acontece la figura 3. 


\section{c. "Con mis hijos no te metas"}

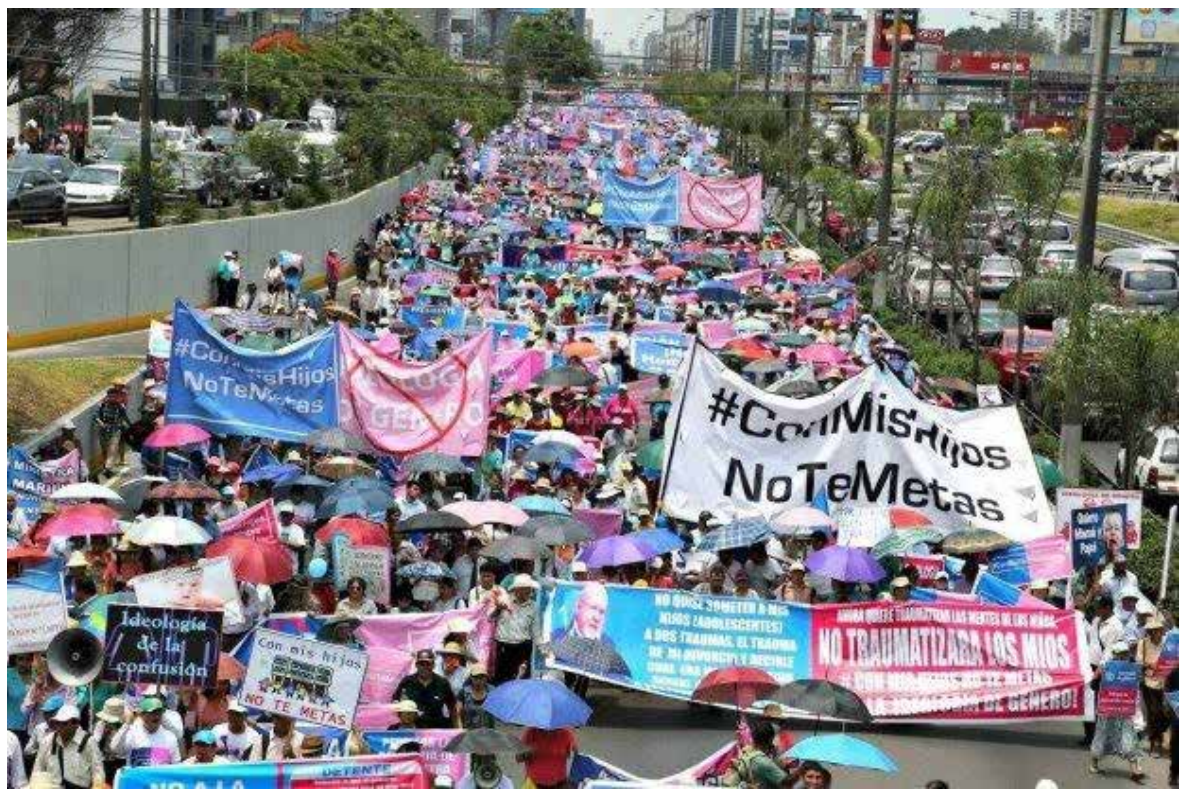

Figura 3. Marcha del Colectivo "Con mis hijos no te metas" inicia a las cuatro de la tarde. Fecha: 4 de marzo de 2017. Fuente: Diario La República.

La figura 3 plasma la movilización realizada el 4 de marzo de 2017 en Lima y que unió a sectores eclesiales evangélicos con católicos y asociaciones de padres afines. Los lemas que rodearon el encuentro puntualizaron en denunciar la intromisión del estado en la educación de niños y niñas conceptualizadxs en el estatus de "hijos". ${ }^{10}$ Entre las consignas se escuchó: "El gobierno quiere homosexualizar a la niñez"; "Ministra, no homosexualices a nuestros hijos"; "Los niños necesitan una familia real"; "Somos colonia del Club de París. PPK nos vendió a cambio de homosexualizar a los niños del Perú". ${ }^{11}$

La acción organizada de estos sectores, que también encontró eco entre figuras políticas como Nelly Cuadros ${ }^{12}$ así como también en las acciones judiciales que lograron algún éxito, ${ }^{1314}$ lograron en el transcurso de 2017 modificar los lineamientos curriculares retirando la palabra "género".

La ideología de género ${ }^{14}$ emerge como concepto de lucha y denuncia para estos sectores renovando articulaciones que antaño no resultaban tan transparentes. La aparición de los sectores evangélicos en alianza con el catolicismo renueva la pregunta sobre cómo esta articulación irrumpe en la región. A su vez, las vinculaciones de estos sectores en distintos estamentos (políticos, jurídicos o en los medios de comunicación) reconfiguran su estrategia y posiciones de poder. En este escenario, distintos autorxs señalan a la fuerza conservadora que avanza sobre conquistas populares como una vertiente potente desde la cual los gobiernos actuales de la región intervienen. En el próximo apartado problematizaré otra vertiente desde la cual parece organizarse la nueva grilla política territorial.
10.El video de intervención de Nelly Cuadros en la Comisión de Educación del Congreso Nacional de Perú se puede encontrar en $<$ https://www. youtube.com/watch?v=g]fxJ/K ${ }_{3} \mathrm{GDpE}>$ El debate social encuentra en este tema un diálogo con algunos sectores políticos que ocupan cargos de representación. En este caso, diputados y diputadas hicieron audibles las demandas del movimiento Ni una menos en sus respectivas cámaras. 11. Selección de notas alusivas: La República, (04, marzo, 2017) 'Con mis hijos no te metas': así se desarrolla la marcha contra la "ideología de género" [FOTOS y VIDEOS]. Recuperado de < http:// larepublica.pe/sociedad/853601larepublica.pe/sociedad/853601-
con-mis-hijos-no-te-metas-manifestantes-se-reunen-para-marcharcontra-la-ideologia-de-genero> (consulta: 19-05-2019).

Perú21, (o4, marzo, 2017) ¿Quiénes apoyan la marcha nacional "Con mis hijos no te metas'? [Nota]. mis hijos no te metas'? [Nota].
Recuperado de < https://peru21. pe/lima/quienes-apoyan-marchanacional-mis-hijos-metas-67174> (consulta: 19-05-2019).

12. Nelly Cuadros es el congresista de la Fuerza Popular por Cusco. 13. Agencia Informativa Católica Argentina, (06, septiembre, 2017) Padres de Familia frenan la ideología de género en las escuelas [Nota]. Recuperado de <http://www.aica. org/30102-padres-de-familia-frenanla-ideologia-genero-en-las-escuelas. html> (consulta: 19-05-2019). 14. Para un rastreo histórico de la categoría en America Latina: Miskolci, R. y Campana, M. (2017). "Ideologia de gênero": notas para a genealogia de um pânico moral contemporâneo. Sociedade e Estado vol. 32 (n ${ }^{\circ}$ ), pp. $725-748$. doi: <https://dx.doi.org/10.1590/ s0102-69922017.3203008>. 


\section{d. Chau Tabú}

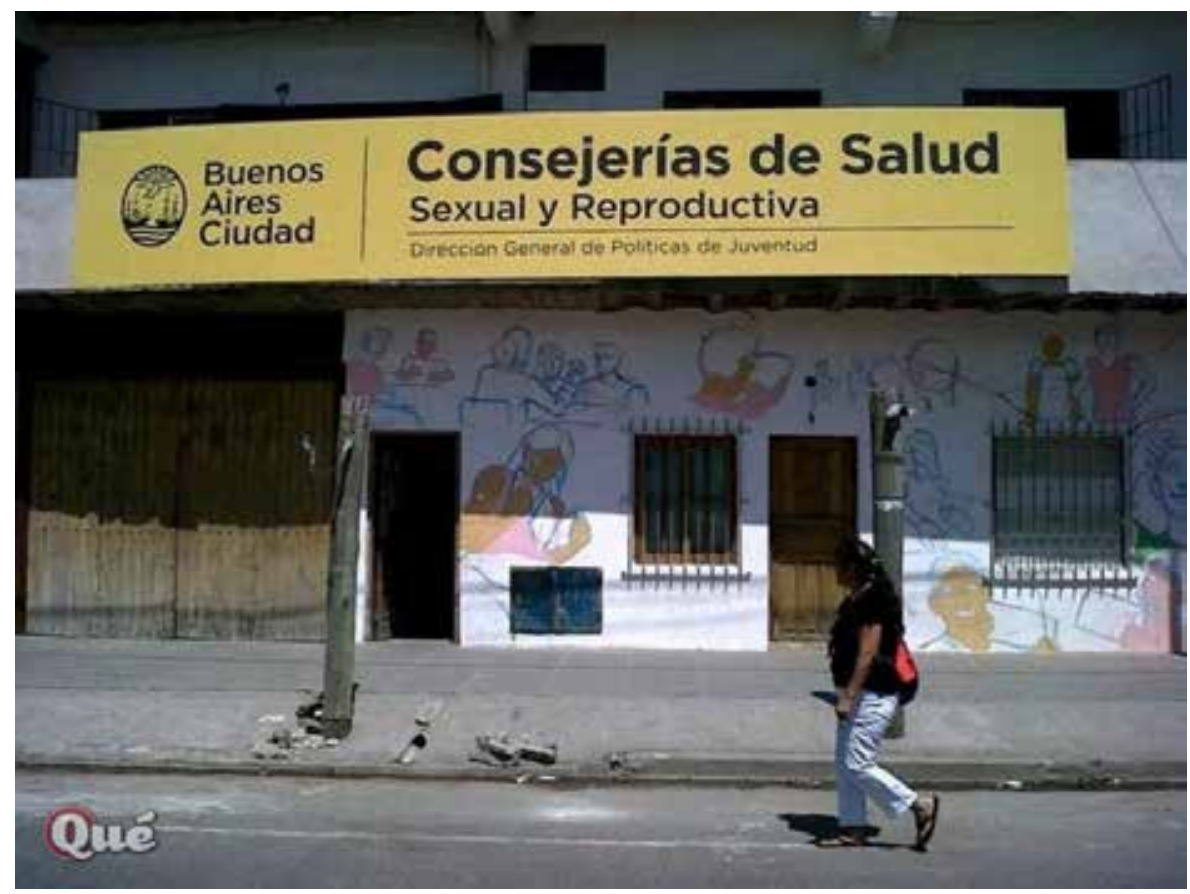

Figura 4. Revista Que. Fecha: 8 de enero de 2018. Fuente: <http:// www.revistaque.com/5/nota.php?nota_id=14759>.

Chau Tabú es un programa presentado desde la jefatura de gobierno como una "herramienta de educación sexual" del gobierno de la Ciudad de Buenos Aires en el marco de la Dirección de Políticas de Juventud, que depende de la vicejefatura de gobierno. Cuenta con el acompañamiento de la Fundación Huésped, la Fundación Lactancia y Maternidad (Fundalam) y la Federación Argentina de Lesbianas, Gays y Transexuales (FALGBT). El programa incluye una página web ${ }^{15}$ en la que aparecen historias, ${ }^{16}$ información, consultorio online, entre otros. Además de consejerías en dos comunas de la Ciudad en las que se distribuían métodos anticonceptivos y se brindaba asesoría. En el mes de diciembre de 2017, el gobierno decidió cerrarlas.

La noticia se encadena a una serie de medidas que avanzan con el desmantelamiento de políticas vinculadas a la educación sexual. En primer lugar, la existencia del programa Chau Tabú expresa una modalidad específica de inclusión de la educación sexual que no depende del Ministerio de Educación sino de una Dirección que depende directamente del jefe de gobierno. Este hecho, fuertemente denunciado por organizaciones de la sociedad (por ejemplo, el Frente Popular por la ESI ${ }^{17}$ se entrelaza con la reducción de recursos -económicos y humanos-a los programas de Educación Sexual propiamente dichos. Sancionada la Ley 2110 de Educación Sexual Integral en la Ciudad de Buenos Aires una serie de acciones comenzaron a configurar el abordaje de la educación sexual en la jurisdicción. Con la llegada del partido Propuesta Republicana-PRO- al gobierno, la acciones ancladas en el campo pedagógico comenzaron a migrar a otras áreas: salud, desarrollo social o política juvenil, construyendo tres apuestas que discuten el sentido de la educación sexual como contenido político pedagógico integral. En esta dirección la ESI aparece como I. asunto de salud, II. asunto de mujeres pobres y III. asunto juvenil. En segundo lugar, el cierre de los espacios de las salas es un signo más sobre el proceso de desmantelamiente ${ }^{18}$ que atravesamos hoy. 
Esta escena abre una serie de interrogantes para la región: ¿Qué desplazamientos, corrimientos o giros atraviesan hoy las políticas públicas vinculadas a la educación sexual? ¿Qué espacio ocupa en la formulación la gramática de la salud? ¿Qué asignación presupuestaria se destina?

\section{A modo de cierre. Configuraciones y actualizaciones políticas}

En la última década, desde una mirada general, la región incluyó en su agenda política la educación sexual desde una mirada integral y en diálogo con otras leyes vinculadas al campo de las sexualidades que reactualizó su letra. Incluso, varios países se vieron interpelados por movimientos como por ejemplo: Ni una menos. Esta nueva interpelación social implicó en la región una reconfiguración de las acciones y discursos en la temática abriendo interrogantes respecto de cómo hoy se trabaja la educación sexual desde una perspectiva feminista. Sin quedar totalmente clarificado cada uno de estos conceptos, el movimiento Ni una menos actualiza al contexto contemporáneo la "violencia machista", "patriarcado", "feminismo", "feminicidio", entre otros, abriendo el debate.

En una coyuntura en la que avanza una conducción política neoliberal - neoconservadora, la marcha Con mis hijos no te metas y Chau tabú nos brindan aristas desde donde anclar dos tendencias que organizan hoy las políticas públicas de la región. Por un lado, potenciando grupos de poder que denuncian la intromisión del estado en las vidas privadas al tiempo que reclaman la restitución de "los padres" como educadores naturales. Por otra parte, el corrimiento del eje de lo educativo a la salud nos advierte sobre un segundo desplazamiento de lo político a una concepción de pretensión especializada y aséptica. Estos nuevos (viejos) sectores encuentran alianzas religiosas y en la estructuras de representación política, así como en la sociedad civil.

Las escenas pedagógicas contemporáneas propuestas tensionan y disputan la arena de sentidos entre la normativización conservadora, la reestructuración neoliberal y las resistencias feministas, abriendo interrogantes sobre la configuración de las tendencias sobre la que avanza la región. 


\section{Q Bibliografía}

» Baez, J. (2015). Políticas educativas, jóvenes y sexualidades en América Latina y el Caribe. Las luchas feministas en la construcción de la agenda pública sobre educación sexual. Informe CLACSO. Recuperado de <http://biblioteca.clacso.edu.ar>.

» Baez, J. y González del Cerro, C. (2015). Políticas de Educación Sexual: tendencias y desafíos en el contexto latinoamericano. Revista del Instituto de Investigaciones en Ciencias de la Educación ( ${ }^{\circ}$ 38), pp. 7-24.

»Batista de Oliveira Júnior, I. (2016). Kit de combate a homofobia do mec: a polemização em torno dos recursos audiovisuais. HISTEDBR On-line ( $\left.n^{\circ} 70\right)$, pp. 319-334.

» Brown, J. (2007) Ciudadanía de mujeres en Argentina. Los derechos (no) reproductivos y sexuales como bisagra, lo público y lo privado puesto en cuestión (Tesis de maestría). FLACSO, Argentina.

"Carosio, A. (Coord.). (2014). Feminismos para un cambio civilizatorio. Caracas, Venezuela: CLACSO.

» Foucault, M. (1977). Historia de la sexualidad. Barcelona, España: Siglo XXI.

»Fraser, N. (2006). Reinventar la justicia en un mundo globalizado. New Left Review $\left(n^{\circ} 36\right), p p .31-50$.

»Felitti, K (2012) La revolución de la píldora. Sexualidad y política en los sesenta. Buenos Aires / Barcelona: Edhasa; 2012, 224 p. ISBN 978-98-. 7628-165-2.

" Lavigne, L. (2011). Las sexualidades juveniles en la educación sexual integral. En Elizalde, S. (Coord.), Jóvenes en cuestión. Configuraciones de género y sexualidad en la cultura. Buenos Aires, Argentina: Biblos.

»Maffia, D. (2001). Ciudadania Sexual. Feminaria, año XIV (n²6).

» Maio, E. R. y Batista de Olivera Júnior, I. (2015). Diversidade sexual e homofobia: a cultura do "desagendamento" nas políticas públicas educacionais. Práxis educativa, vol.10 ( $\mathrm{n}^{\circ}$ 1), pp.35-53. doi: <10.5212/PraxEduc.v.10i1.0002>.

" Ministerio de Educación de la República del Perú. (2016). Currículo Nacional de la educación básica. Recuperado de <http://www.minedu.gob.pe/curriculo/pdf/ curriculo-nacional-2016-2.pdf>.

»Ministerio de Educación de la República del Perú. (2008). Lineamientos educativos y orientaciones pedagógicas para la Educación Sexual Integral. Manual para profesores y tutores de Educación Básica Regular. Recuperado de <http://bvs. minsa.gob.pe/local/minsa/1283_gob523.pdfs.

»Morgade, G. (2006). Sexualidad y prevención: discursos sexistas y heteronormativos en la escuela media. Revista del Instituto de Investigaciones en Ciencias de la Educación ( $\left.\mathrm{n}^{\circ} 24\right)$, pp. 27-33.

»Morgade, G. (Coord.). (2011). Toda educación es sexual. Hacia una educación sexuada justa. Buenos Aires, Argentina: Crujía Ediciones.

»Sagot, M. (2014). La democracia en su laberinto: el neoliberalismo y los límites de la acción política feminista en Centroamérica. En Carosio, A. (Coord.), Feminismos para un cambio civilizatorio. Caracas, Venezuela: CLACSO.

"Sontag, S (2003) La enfermedad y sus metáforas - el sida y sus metáforas. Buenos Aires. Taurus 